\title{
Caso de EVC isquémico y tromboembolia pulmonar asociado a foramen ovale permeable
}

Tomás Miranda-Aquino*, Silvia E Pérez-Topete*.

*Residentes de Medicina Interna 3er año, Hospital Christus Muguerza Alta Especialidad/UDEM.

Copyright (C) 2016 por autores (s) y Revista Biomédica.

Este trabajo esta licenciado bajo las atribuciones de la Creative Commons (CC BY).

http://creativecommons.org/licenses/by/4.0/

(c) (i) Open Access 


\title{
Resumen
}

Introducción: Caso clínico: Se presenta el caso de una paciente femenina de 53 años, con antecedente de una safenectomía 2 días previos a su ingreso. Ingresa a urgencias por cuadro de pérdida del estado de alerta súbito, Glasgow 3, por lo que se realiza intubación orotraqueal, presenta hipotensión persistente que se maneja con vasopresores. Se le realiza AngioTAC cerebral y se diagnostica EVC isquémico, es ingresado a terapia endovascular, durante el procedimiento presenta desaturación persistente, a pesar de aporte de $\mathrm{O} 2$ al 100, motivo por el cual se realiza Angio TAC pulmonar y se le diagnostica tromboembolia pulmonar, se realiza EcoCardiograma transtorácico el cual reporta foramen ovale permeable. Paciente posterior presenta pérdida de reflejos de tallo, se realiza TAC de cráneo que reporta conversión hemorrágica. Se diagnostica muerte cerebral un día después y se ingresa a protocolo de trasplante. Entra a cirugía de donación de órganos donde se procuran riñones, hígado, hueso y córneas.

Palabras Clave: Foramen ovale, Tromboembolia pulmonar, EVC isquémico.

\begin{abstract}
Female patient 53 years old, presented with a history of saphenectomy two days before admission. Upon arrival to the emergency room because sudden loss of status alert, Glasgow 3, endotracheal intubation is done, she had persistent hypotension with vasopressors handled. She is performed CT angiography and cerebral ischemic stroke is diagnosed, she is entered endovascular therapy, during the procedure have persistent desaturation, despite $\mathrm{O} 2$ supply $100 \%$, pulmonary $\mathrm{CT}$ angiography was performed and was diagnosed with pulmonary embolism, transesophageal echocardiogram is made which reports patent foramen ovale. 6 hours later patient has lost stem reflexes, cranial CT is performed reporting hemorrhagic brain conversion. It is diagnosed brain dead a day later and is entered transplant protocol. Enter organ donation surgery where kidneys, liver, bone and corneas were procurement.
\end{abstract}

Keywords: Foramen ovale, Pulmonary embolism, Ischemic stroke.

\section{Introducción}

El foramen oval es una división interauricular dentro del septum secundum, que sirve como un conducto entre las aurículas derecha e izquierda (1). Esta comunicación persistente podría permitir que la sangre desoxigenada y los émbolos puedan pasar, paradójicamente, desde la circulación derecha a izquierda1.

El embolismo paradójico se define como un émbolo que entra a la circulación arterial sistémica desde trombos venosos a través de una derivación de derecha a izquierda. La derivación de derecha a izquierda más común, es el foramen ovale permeable (2).

El foramen ovale permeable se encuentra en $25 \%$ de la población; se cree que hasta $15 \%$ de los pacientes con EVC isquémico criptogénico son debidos a foramen ovale permeable3.

\section{Caso clínico}

Paciente femenina de 53 años de edad, sin antecedentes médicos de importancia. Es sometida a safenectomía izquierda dos días previos a su ingreso. Es egresada el mismo día de la intervención quirúrgica solamente con AINES como parte del manejo médico. 
La paciente inicia el padecimiento actual media hora previa a su ingreso al ser encontrada inconsciente sin respuesta a ningún estímulo. Los familiares refieren que 2 horas previas había sido la última vez que se encontraba alerta y completamente asintomática. Se trasladó al servicio de urgencias donde se encontraron los siguientes signos vitales: TA: 70/50mmHg, FC: 105 lpm, FR: 22 rpm, Temp: $35.6^{\circ} \mathrm{C}$, Sat O2: 69\%. A la exploración física se encontró a la paciente en estado de coma, con puntaje Glasgow de 3, pupilas anisocóricas, con reflejo fotomotor y consensual presente. Ruidos cardiacos rítmicos, sin soplos, sin ruidos agregados. Pulmones con discretos estertores roncantes bibasales. Abdomen asignológico. Extremidades cianóticas, frías, pulsos débiles; miembro pélvico inferior izquierdo con herida quirúrgica limpia, con leve eritema.

La biometría hemática reportó $\mathrm{Hb}: 12.1$ g/dL, VCM: 90.7, CMCH: 31.2, Leucocitos: 11900, Neutrófilos: 4200, Linfocitos: 6900, Monocitos: 500, Eosinófilos: 130, Basófilos: 0. En el perfil bioquímico se encontró lo siguiente: Glucosa: $483 \mathrm{mg} / \mathrm{dL}$, Creatinina: $1.1 \mathrm{~g} / \mathrm{dL}$, BUN: 12.5, Urea: 26.8, Ácido úrico: 5.2, Colesterol: 214, Trigliceridos 227, Bilirrubina directa: 0.2, Bilirrubina indirecta: 0.3, Albúmina: 3.7, Globulina 2.7, AST: 28, ALT: 34, Fosfatasa alcalina: 73, DHL: 286, Na: 135, K: 3.13, Cl: 90.7, Ca: 9.1, PO: 7.3. La medición de gases arteriales arrojó los siguientes datos: pH: 7.05, pCO2: 62, pO2: 74, HCO3: 17.2, Sat: 60\%, Lac: 9.2. Los tiempos de coagulación resultaron como sigue: TP: 16.1, INR: 1.17, TTP: 39.7.

Debido al estado de consciencia se realiza intubación orotraqueal. Se inicia soporte hemodinámico mediante la administración de 1500 cc de Hartman a goteo rápido sin obtenerse respuesta, por lo que se inicia Dopamina. 20 minutos después de su ingreso se le realiza TAC de cráneo simple (Imagen 1), el cual reporta que no hay evidencia de eventos hemorrágicos ni isquémicos. Se hace el diagnóstico de EVC isquémico, motivo por el cual se interconsulta a neurología el cual indica, 1 hora posterior a su ingreso, alteplase $6 \mathrm{mg}$ IV en bolo y $54 \mathrm{mg}$ para 1 hora. Al terminar se realizó una angio-TAC cerebral (imagen 2) que permite observar una región hipodensa hacia la región talámica y globo pálido izquierdo que pudiera corresponder a una zona de isquemia. Dos horas y media después de su ingreso persistió en el mismo estado neurológico, motivo por el cual pasa a realización de trombectomía de arteria cerebral posterior más trombolisis supraselectiva con Alteplase $2 \mathrm{mg}$ dosis única. Durante el procedimiento, la paciente permaneció con desaturación persistente, pese a tener $\mathrm{FiO} 2$ al $100 \%$. Al terminar el procedimiento pasó a la Unidad de Cuidados Intensivos (UCI). A su llegada a UCI tenía pupilas isocóricas con reflejo fotomotor y consensual presente, reflejos de tallo presentes. La paciente continuó hemodinamicamente con tendencia a la hipotensión por lo que se tuvo que iniciar Norepinefrina, con mejoría parcial. Al persistir la desaturación, se sospechó tromboembolia pulmonar (TEP), por lo que se realizó EcoCardiograma transtorácico para buscar datos indirectos de TEP, el cual reportó dilatación del ventrículo derecho, hipoquinesia de la pared libre del ventrículo derecho, FEVI $60 \%$, presión sistólica del ventrículo derecho $28 \mathrm{mmHg}$. Posterior a esto se le realizó un Eco Doppler de miembros inferiores que reportó imagen sugestiva de trombo en la emergencia de la vena safena izquierda. A continuación, se realizó un angio-TAC pulmonar (imagen 3) que resultó positivo para TEP. Con la sospecha de Foramen Ovale permeable se realizó ecocardiograma trans-esofágico siendo positivo para ésta condición. Con base en los hallazgos, 10 horas posteriores a su ingreso, se decide anticoagulación con enoxaparina $60 \mathrm{mg} \mathrm{SC} \mathrm{c/12} \mathrm{horas.}$

Tras doce horas de su ingreo, la paciente se encontró hemodinamicamente estable c, e con Sat O2: $>90 \%$ bajo ventilación mecánica, buen control glucémico, sin cambios neurológicos. A las 18 horas de su ingreso presentó inestabilidad hemodinámica, caracterizada por taquicardia y bradicardia intermitente, además de hipotensión persistente, por lo que se agregó vasopresina. Además, al momento de estos cambios hemodinámicos se encontró con pupilas anisocóricas, sin respuesta a la 
luz y ausencia de los reflejos de tallo. Se le toma TAC de cráneo simple (imagen 4) de urgencia, resultando con datos en relación a sangrado difuso asociado a herniación de amígdalas cerebelosas.

Tras 24 horas de su ingreso se le realizó electroencefalograma, que no reportó actividad eléctrica cortical; así como un estudio de potenciales evocados auditivos y visuales que no mostraron respuesta. A las 48 horas se repite el electroencefalograma que no reporta actividad cortical, con lo que se diagnostica muerte cerebral. Finalmente, la paciente entra en protocolo de trasplante renal, a través de una cirugía de donación de órganos donde se procuraron riñones, hígado, hueso y córneas.

\section{Discusión}

Cerca del $40 \%$ de los EVC isquémicos no tienen una causa identificable y se clasifican como criptogénicos4. El riesgo anual de embolismo paradójico en pacientes con foramen ovale permeable es de 28 por cada 100000 personas5.

La paciente de nuestro caso ingresó con un diagnóstico de EVC isquémico, y como todo paciente que ingresa con este diagnóstico presuntivo se debe realizar TAC de cráneo simple como primer estudio de imagen6. Al resultar negativo para hemorragia, fue tratada como se menciona en la literatura6 mediante terapia de trombolisis sistémica, ya que se encontró en ventana terapéutica de 3 horas, utilizando una dosis recomendada de $0.9 \mathrm{mg} / \mathrm{kg}$ de alteplase, dando el $10 \%$ en bolo y el resto para 1 hora. Como lo menciona Singh et al7 existe la terapia endovascular, la cual ha demostrado ser efectiva para pacientes con NIHSS mayor de 20, que a pesar de haber demostrado una tasa de retrombosis del $34 \%$ contra $17 \%$ de la terapia endovascular, aún existe controversia sobre cuál es superior 8 .

La paciente se le diagnosticó posteriormente TEP, que según la estratificación de riesgo por la escala PESI fue de V. La estratificación depende de criterios clínicos, la determinación de biomarcadores cardiacos (troponina) y la estimación de la función y anatomía del ventrículo derecho por ecocardiograma9. Otra manera de estratificar es en masivo, submasivo y de bajo riesgo. Se considera masivo aquellos que se presentan con inestabilidad hemodinámica; y se considera submasivo aquellos que se encuentran hemodinamicamente estables, pero con biomarcadores cardiacos positivos o datos de disfunción del ventrículo derecho10. El método de imagen de elección para detectar TEP actualmente es el angio-TAC pulmonar11, tal y como se le realizó a nuestra paciente, teniendo una sensibilidad del $97 \%$ y una especificidad del $98 \% 10$.

El tratamiento dependerá del estado hemodinámico del paciente. En pacientes inestables está indicada la trombolisis sistémica o la embolectomía, al igual que en pacientes que se encuentran hemodinamicamente estables, pero con datos de disfunción del ventrículo derecho; en pacientes que se encuentran hemodinamicamente estables, sin datos de disfunción del ventrículo derecho, pero con biomarcadores cardiacos positivos existe controversia de si dar trombolisis sistémica o anticoagulación solamente. En los pacientes estables, sin disfunción del ventrículo derecho ni biomarcadores positivos, se recomienda solamente la anticoagulación10. La paciente del caso presentado se encontró hemodinamicamente inestable y con datos de disfunción del ventrículo derecho; por lo que, aunque ameritaba trombolisis sistémica, debido al riesgo de conversión hemorrágica del EVC isquémico se optó por la anticoagulación. La paciente presentó conversión hemorrágica y se diagnosticó muerte cerebral, la cual se define como la ausencia de reflejos de tallo, respuesta motora, y respuesta ventilatoria en un paciente normotérmico, sin sedación, comatoso; con una causa irreversible de daño cerebral y sin causas metabólicas que contribuyan al estado12. En conclusión, se presentó una paciente con EVC isquémico criptogénico, asociado con Foramen Ovale Permeable, coexistiendo con tromboembolismo pulmonar. Es importante bajo la presencia de esta condición, sospechar y tratar precozmente dichas patologías, para disminuir su morbimortalidad. 


\section{Referencias}

1. Kanaganayagam GS, Malik IS. Modern management of a patent foramen ovale. J R Soc Med Cardiovasc Dis. 2012; 1: 19.

2. Park MS, Park JP, Yun SH, Lee JU, Kim JK, et al. A Case of Cryptogenic Stroke Associated with Patent Foramen Ovale Coexisting with Pulmonary Embolisms, Deep Vein Thromboses, and Renal Artery Infarctions. Korean Circ J 2012; 42: 853-856.

3. Mirzada N, Ladenvall P, Hansson PO, Eriksson P, Dellborg M. Multidisciplinary management of patent foramen ovale and cryptogenic stroke/TIA. Journal of Multidisciplinary Health Care. 2013; 6: 357-363.

4. Elmariah S, Furlan AJ, Reisman M, Burke D, Vardi M, et al. Predictors of Recurrent Events in Patients with Cryptogenic Stroke and Patent Foramen Ovale Within the CLOSURE I Trial. JACC: Cardiovascular Interventions. 2014; 7 (8): 913-20.

5. Meier B, Frank B, Wahl A, Diener HC. Secondary stroke prevention: patent foramen ovale, aortic plaque, and carotid stenosis. European Heart Journal. 2012; 33: 705-713.

6. Jauch E, Saber J, et al. Guidelines for the Early Management of Patients With Acute Ischemic Stroke: A Guideline for Healthcare Professionals From the American Heart Association/American Stroke Association. Stroke. 2013; 44: 870-947.

7. Singh B, Parsaik A. Endovascular Therapy for Acute Ischemic Stroke: A Systematic Review and Meta-analysis. Mayo Clin Proc. 2013; 88 (10):1056-1065.

8. Falluji N, Abou-Chebl A. Reperfusion Strategies for Acute Ischemic Stroke. Angiology. 2012 april; 63(4) 28996.

9. Goldhaber SZ. Advanced treatment strategies for acute pulmonary embolism, including thrombolysis and embolectomy. J Throm and Haemost. 2009; 7(1): 322-7.

10. Piazza G, Goldhaber S. Fibrinolysis for acute pulmonary embolism. Vascular Medicine. 2010; 15(5): 419-428.

11. Huisman MV, Klok FA. Diagnostic management of acute deep vein thrombosis and pulmonary embolism. 2013; 11: 412-422. 


\section{Apéndices}

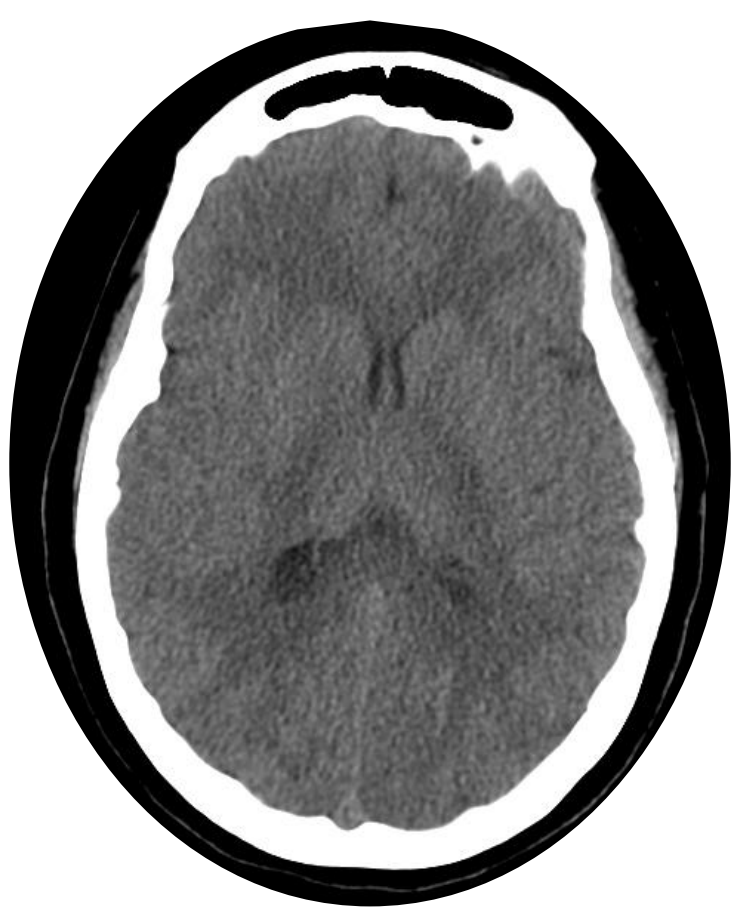

Imagen 1: TAC de cráneo simple que demuestra la ausencia de eventos isquémicos y hemorrágicos.

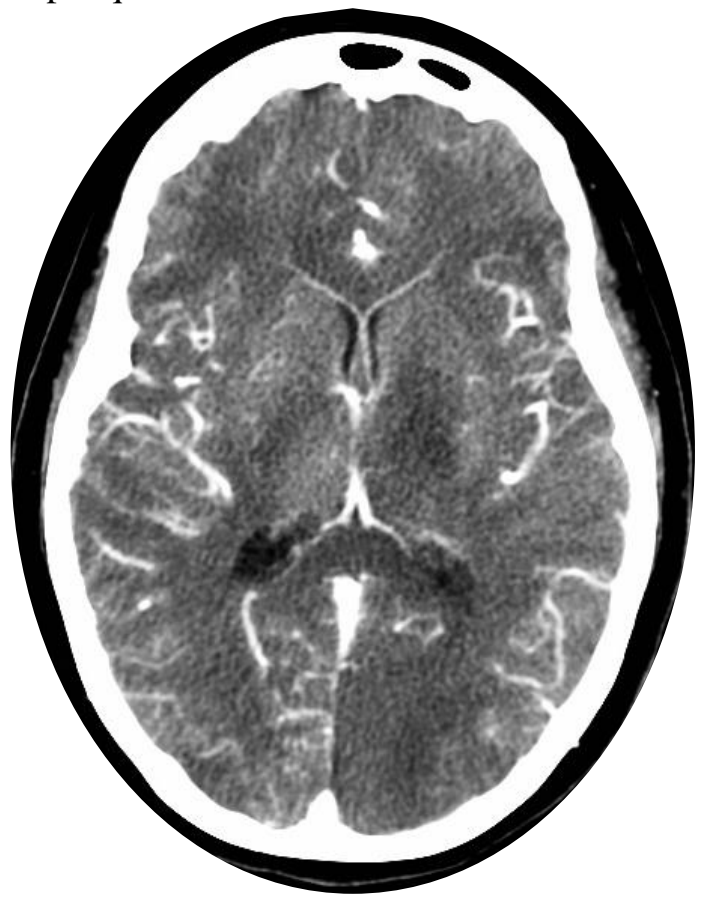

Imagen 2: AngioTAC cerebral que demuestra zona hipodensa hacia la región talámica y globo pálido izquierdo que pudiera corresponder a zona de isquemia. 


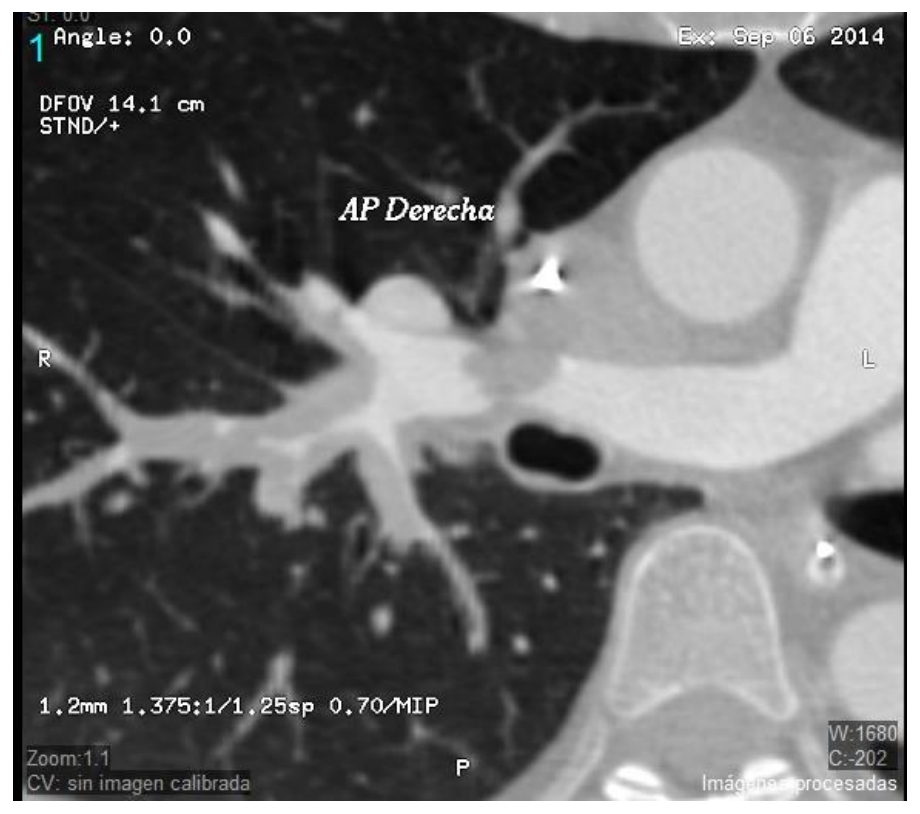

Imagen 3: AngioTAC pulmonar que demuestra trombosis de la arteria pulmonar derecha de primero y segundo orden.

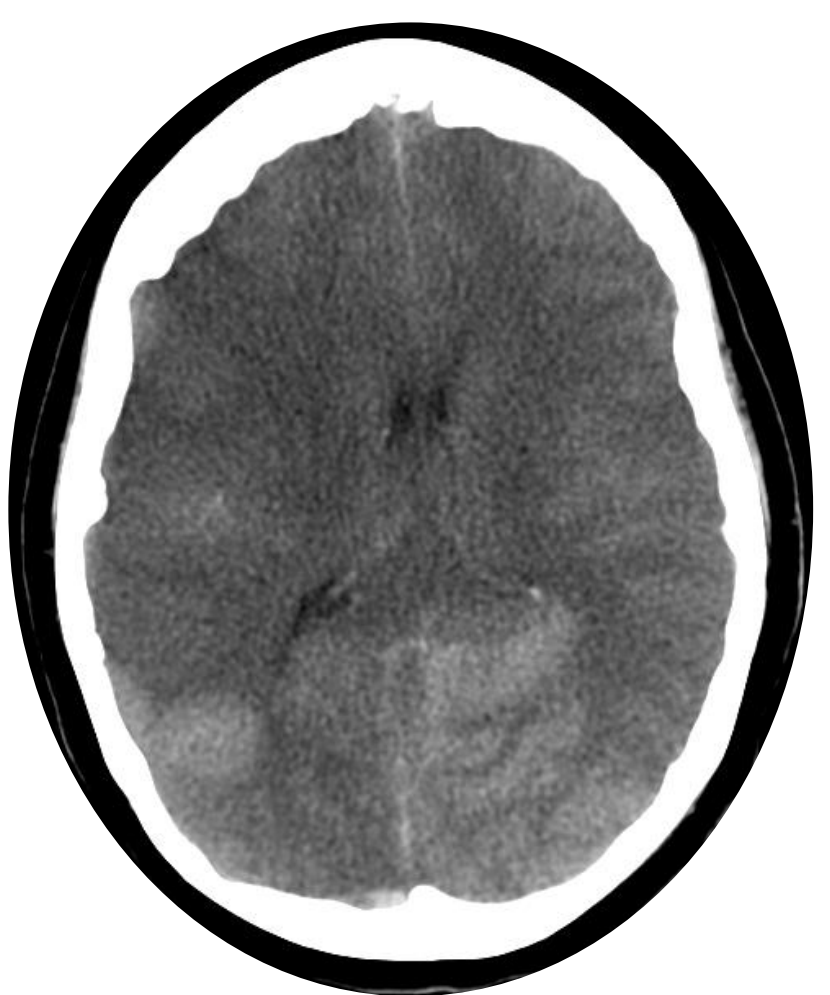

Imagen 4:TAC de cerebro simple que demuestra borramiento de surcos y circunvoluciones, se observan múltiples áreas de hipodensidad de forma difusa de predominio hacia el hemisferio derecho así como imágenes hiperdensas debido a sangrado parenquimatoso de predominio hacia el lóbulo parietal derecho y ambos lóbulos occipitales. 